\title{
Metodología para la medición de la productividad en instituciones prestadoras de servicios de salud*
}

\author{
[Artículo de revisión]
}

\author{
Tatiana Carolina Cadavid Hincapié** \\ Hernán Augusto Gómez Gómez ${ }^{* * *}$ \\ Harold Wilson Hernández Cruz ${ }^{* * * *}$
}

Recibido: 7 de noviembre de 2020

Revisado: 12 de enero de 2021

Aceptado: 14 de marzo de 2021

Citar como:

Cadavid Hincapié, T. C., Gómez Gómez, H. A. y Hernández Cruz, H. W. (2021). Metodología para la medición de la productividad en instituciones prestadoras de servicios de salud. Signos, Investigación en Sistemas de Gestión, 13(2). https://doi.org/10.15332/24631140.6670

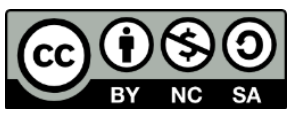

\footnotetext{
*Artículo de revisión.

** Bacterióloga. Línea de investigación en calidad y gestión integral. Universidad Santo Tomas, convenio Icontec, Bogotá, Colombia. Correo electrónico: tatianacadavid@usantotomas.edu.co, carito7834@hotmail.com; ORCID: https://orcid.org/0000-0003-2636-3566; CvLAC: https://scienti.minciencias.gov.co/cvlac/visualizador/generarCurriculoCv.do?cod rh=0001488331 ${ }_{* * *}$ Especialista en auditoría en salud y enfermero. Línea de investigación en calidad y gestión integral. Universidad Santo Tomas convenio Icontec, Bogotá, Colombia. Correo electrónico: hernangomezg@usantotomas.edu.co, augomez79@yahoo.com; ORCID: https://orcid.org/00000001-8227-2592. CVLAC:

https://scienti.minciencias.gov.co/cvlac/visualizador/generarCurriculoCv.do?cod rh=0001776061 ${ }^{* * * *}$ Magíster en Ciencias de la Educación, especialista en Educación Mediada por TIC e ingeniero industrial por la Universidad Santo Tomás. Línea de investigación en calidad y gestión integral. Correo electrónico: harold.hernandez@usantotomas.edu.co; ORCID: https://orcid.org/00000002-9374-6703. CVLAC:

https://scienti.minciencias.gov.co/cvlac/visualizador/generarCurriculoCv.do?cod rh=0001592345
}

Signos, Investigación en Sistemas de Gestión

ISSN: 2145-1389 | e-ISSN: 2463-1140 | DOI: https://doi.org/10.15332/24631140

Vol. 13 N.o 2 | julio-diciembre de 2021 


\section{Resumen}

Se presenta un análisis de las diferentes metodologías descritas a nivel mundial para la medición de la productividad en el sector de la salud. Para ello, se desarrolla una revisión bibliográfica descriptiva a partir de literatura encontrada en bases de datos indexados y búsqueda iterativa. Como resultados, se encontró que las principales metodologías empleadas actualmente para la medición de la productividad y eficiencia son el análisis envolvente de datos (DEA) en combinación con otros métodos. Finalmente, se concluye que son varias las metodologías de medición descritas en la literatura y en cada una de ellas se encuentra la combinación de diferentes métodos y variables a evaluar, de acuerdo con el enfoque del investigador; sin embargo, se hace necesario el desarrollo de estudios adicionales en los que se aborden nuevas variables que influyen en el crecimiento y decrecimiento de la eficiencia y productividad, así mismo, su relación con la calidad en la prestación de los servicios en salud.

Palabras clave: salud, productividad, medición, malmquist, DEA.

\section{Methodology for measuring productivity in health services providers institutions}

\section{Abstract}

An analysis of the different methodologies described worldwide for measuring productivity in the health sector is presented. For this, a descriptive literature review is developed from the literature found in indexed databases and an iterative search. As results, it was found that the main methodologies currently used for measuring productivity and efficiency are data envelopment analysis (DEA) in combination with other methods. Finally, it is concluded that there are several measurement methodologies described in the literature and in each one of them there is a combination of different methods and variables to be evaluated, according to the researcher's approach; However, developing 
further studies is necessary to address new variables that influence the growth and decrease of efficiency and productivity, as well as their relationship with quality in the provision of health services.

Keywords: health, productivity, measurement, malmquist, DEA.

\section{Metodologia para medir a produtividade em instituições fornecedoras de serviços de saúde.}

\section{Resumo}

É apresentada uma análise das diferentes metodologias descritas no nível mundial para a medição da produtividade no setor da saúde. Para isso, uma revisão descritiva da bibliografia é feita com base na bibliografia encontrada em bancos de dados indexados e pesquisa iterativa. Como resultado, verificou-se que as principais metodologias atualmente utilizadas para a medição da produtividade e eficiência são a Análise de Envoltória de Dados (DEA), em combinação com outros métodos. Finalmente, conclui-se que existem várias metodologias de medição descritas na bibiografia havendo em cada uma delas uma combinação de diferentes métodos e variáveis a serem avaliadas, conforme abordagem do pesquisador; Contudo, são necessários estudos adicionais que abordem novas variáveis que influenciam o crescimento e diminuição da eficiência e produtividade, bem como sua relação com a qualidade na prestação de serviços de saúde.

Palavras-chave: saúde, produtividade, medição, malmquist, DEA.

\section{Introducción}

El desarrollo de herramientas metodológicas para la medición de la productividad en el sector de la salud ha mostrado ser una de las líneas de investigación actual. Esto debido a que el funcionamiento adecuado de los servicios sanitarios se encuentra dentro de las grandes preocupaciones que enfrenta la sociedad. En este confluyen dos actores o puntos de vista; por 
un lado, encontramos a los pacientes, quienes desean recibir atención que resulte satisfactoria y de calidad; y, por el otro, encontramos a los contribuyentes, quienes desean una asignación optima de los recursos, mediante la cual puedan garantizar una adecuada prestación de servicios en salud, según lo plantea Díaz y Gómez (2013). Por ende, la medición de la productividad en dicho ámbito resulta ser un tema de gran interés y utilidad, tanto para las personas que demandan los servicios, como para las instituciones prestadoras de los mismos; esto basándose en la premisa de que la productividad mejorará cuando se logren mayores beneficios y mejor atención a la requerida por los usuarios con la menor o igual utilización de recursos.

En este sentido, autores como Prokopenko (1989) definen la productividad como la relación directa entre insumos y productos obtenidos, en la cual se busca lograr el resultado deseado al emplear un menor tiempo de producción. Otro autor, Luna Cardozo (2011) relaciona la productividad de las organizaciones con la calidad ofrecida, el alto desempeño, la gestión estratégica y la mirada social para la provisión de más y mejores resultados; así, se aproximan al concepto de productividad en el sector de la salud que propone Parra (2012). Este concepto no debe entenderse como unidades o productos manufacturados, sino como eventos de servicios efectivos y resultados esperados, por tanto, debe ser concebido como una estructura construida desde el demandante (paciente) hacia un sistema organizacional con base en el conocimiento de sus necesidades. En este marco, características como la intangibilidad, interactividad y naturaleza social, propias de las organizaciones prestadoras de servicios de salud, hacen que la medición de la productividad en este sector no sea sencilla de delimitar. Díaz y Gómez (2013) proponen que para lograr este proceso se debe evaluar la productividad en los diferentes niveles de las organizaciones con distintas entradas y salidas, con el fin de identificar las 
diferentes fases en cada uno de los procesos que se generan en la prestación de los diferentes servicios de salud.

$\mathrm{Al}$ respecto, se han presentado diversos avances, entre los que se destacan los de Djellal y Gallouj (2013), quienes establecen una clasificación de los métodos de medición de la productividad en el sector en dos grupos; el primero recopila aquellos basados en índices (índice de Malmquist e indicador de Luenberger) y el segundo los métodos de medición econométricos, como el análisis envolvente de datos (DEA), como muestran los trabajos de investigación desarrollados por Herrero Tabanera et ál. (2015).

Algunos estudios más recientes utilizan metodologías de medición de la eficiencia y productividad basada en la conjunción de ambos métodos, el índice de Malmquist y el DEA. Así lo describen Gómez Gallego et ál. (2019), en un estudio en el cual se evaluó y analizó la eficiencia técnica en la gestión del sistema de salud de 28 países de la Unión Europea. Por otro lado, Berhe et ál. (2017) proponen un modelo de medición de la productividad denominado productividad invertida válidamente, por medio de una ecuación en la cual se tiene en cuenta la existencia y alcance de los insumos ineficaces y productos no conformes, que no son tenidos en cuenta en la relación producto/insumo utilizada de forma convencional.

De acuerdo a lo mencionado, el análisis y descripción de las metodologías utilizadas para la medición de la productividad en los servicios de salud representa un avance para el sector, ya que con ello se contribuye a dar solución a la problemática actual que atraviesan las instituciones sanitarias y que se traduce en la inadecuada prestación de servicios, insatisfacción de pacientes, disminución en la oferta y crisis económica. Esto proporciona un marco de referencia mediante el cual las organizaciones identifican las diferentes dimensiones que se pueden 
abarcar en el campo sanitario para la medición de productividad; así mismo, identificar las variables más relevantes para su medición que conlleven a las entidades a definir su estado actual y tomar medidas oportunas para el logro de resultados y proyección futura.

Teniendo en cuenta lo anterior, se presenta un análisis de las diferentes metodologías y avances frente a la medición de la productividad en las organizaciones del sector de la salud, basado en una revisión bibliográfica que permitió identificar las siguientes variables técnicas a tener en cuenta: la conceptualización de la eficiencia y la productividad, las entradas y salidas empleadas para la medición de la productividad, las dimensiones abordadas en el sector de la salud para la medición de la productividad y las metodologías de medición de la productividad en el ámbito sanitario.

\section{Metodología}

El proceso de revisión de literatura científica se llevó a cabo con la ayuda de bases de datos multidisciplinarias como (Science Direct, Pubmed, Web of Science, Taylor and Francis online, Scopus, Embase), para lo cual se utilizaron las siguientes palabras clave teniendo en cuenta el objetivo del artículo, "health systems", "measurement", "productivity", "Malmquist" y "DEA". A partir de lo anterior, se definieron las siguientes ecuaciones con el fin de incrementar la especificidad de la búsqueda bibliográfica con sus respectivos operadores booleanos, en las cuales se incluyen todos los términos seleccionados (productivity AND "health services"), (measurement AND productivity AND "health services"), (measuring productivity AND "health services"), y (Malmquist OR DEA AND health AND productivity). Para la formulación de las ecuaciones se siguió este orden (ver figura 1): 
Figura 1. Formulación de ecuaciones para búsqueda de literatura científica

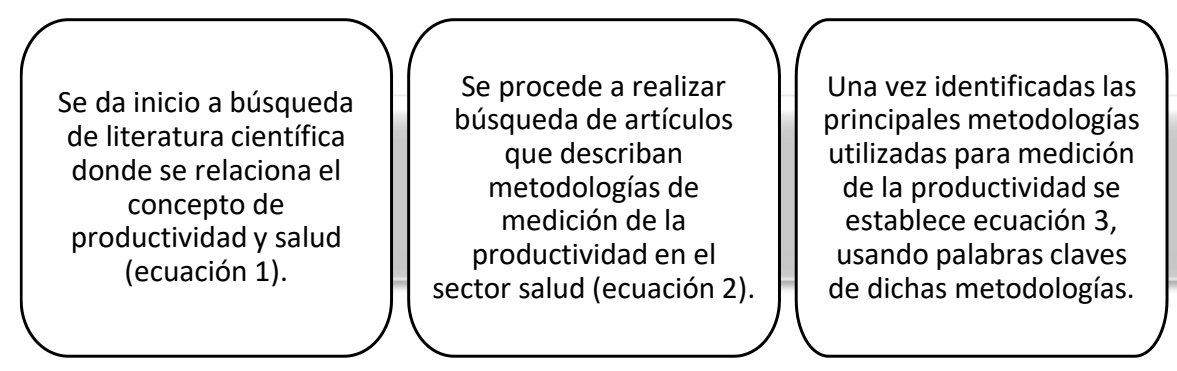

Fuente: elaboración propia.

Las rutas específicas de búsqueda empleadas se describen a continuación:

- $\quad$ Science Direct: productivity AND "health services", measurement AND productivity AND "health services", Malmquist OR DEA AND health AND productivity.

- $\quad$ Pubmed: productivity AND "health services", measuring productivity AND "health services", Malmquist OR DEA AND health AND productivity.

- Web of Science: productivity AND "health services", measurement AND productivity AND "health services", Malmquist OR DEA AND health AND productivity.

- Taylor and Francis online: productivity AND "health services", measurement AND productivity AND "health services", Malmquist OR DEA AND health AND productivity.

- Embase: productivity AND "health services", measurement AND productivity AND "health services", Malmquist OR DEA AND health AND productivity.

- SciELO: productivity AND "health services", measurement AND productivity AND "health services", Malmquist OR DEA AND health AND productivity. 
- Scopus: productivity AND "health services”, measurement AND productivity AND “health services”, Malmquist OR DEA AND health AND productivity.

Los artículos seleccionados se exportaron a Zotero para la eliminación de citas duplicadas y la elaboración de referencias bibliográficas.

\section{Criterios de inclusión y exclusión}

En la búsqueda de literatura científica se incluyeron artículos originales obtenidos en bases de datos científicas indexadas en idioma inglés, entre los años 2015- 2020 o 2015- 2021, de acuerdo con la fuente. En estos se describían las metodologías utilizadas para la medición de la productividad en el sector salud, así, se excluyeron artículos los cuales, posterior a lectura y análisis del resumen, no estaban enfocados en describir las metodologías de medición de la productividad y sus diferentes dimensiones. De este modo se hace énfasis en los métodos utilizados y las variables empleadas para lograr dicho objetivo.

En la búsqueda iterativa en idioma español, se tuvo como único criterio de exclusión que el enfoque de los artículos no fuera el área de la salud y no se describiera la metodología utilizada para la medición de la productividad. 


\section{Resultados de la búsqueda}

Figura 2. Diagrama de flujo depuración de datos

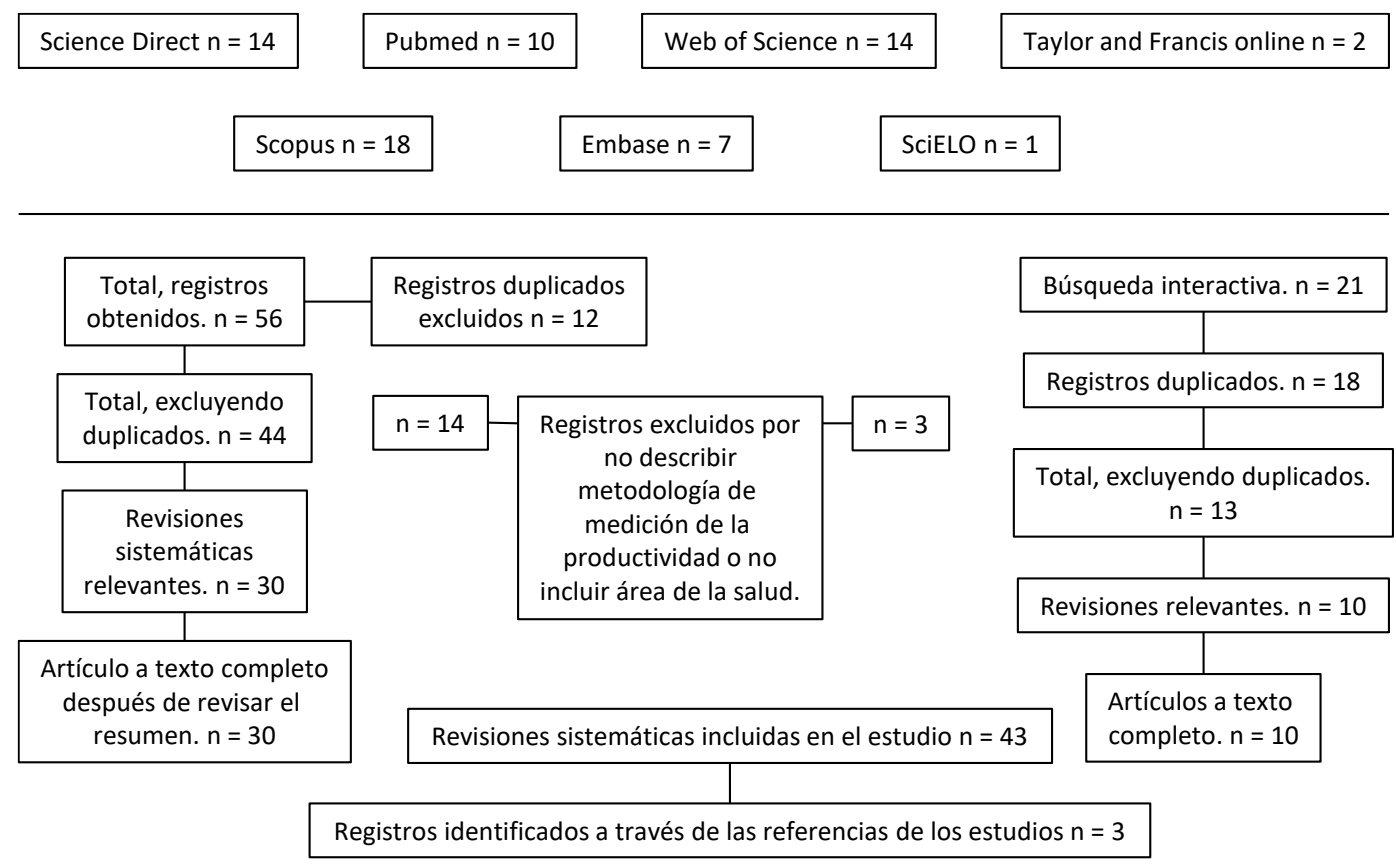

Fuente: elaboración propia (2020).

Los 43 artículos detectados se analizaron y se consideraron variables bibliométricas como año de publicación y país, además, se describieron cualitativamente las siguientes variables técnicas: conceptualización de eficiencia y productividad, entradas y salidas empleadas para la medición de la productividad, dimensiones abordadas en el sector de la salud para la medición de la productividad y metodologías de medición de la productividad en el ámbito sanitario (ver figura 2).

\section{Resultados y discusión}

De los artículos seleccionados para el estudio, se realiza el análisis por año de publicación y país productor de literatura, y se encontró que el mayor número de artículos seleccionados corresponde a publicaciones realizadas en Colombia (13.9 \%), seguido de china (9.3 \%), España (9,3\%) y demás; 
en el análisis por año de publicación también se encontró que el 75 \% de los artículos seleccionados fue publicado en los últimos 6 años, el $15.9 \%$ fue publicado desde el año 2010 al 2014 y el $9.0 \%$ en años anteriores al 2010, tal como se muestra en la tabla 1.

Tabla 1. Porcentaje de artículos seleccionados para el estudio de acuerdo con el país de producción y año publicación

\begin{tabular}{|c|c|c|c|}
\hline País & No artículos & Porcentaje & Años de publicación \\
\hline Colombia & 6 & $13.9 \%$ & $\begin{array}{c}2012 \text { (2), } 2014,2017,2018, \\
2019 .\end{array}$ \\
\hline China & 4 & $9.3 \%$ & 2017(2), 2019 (2). \\
\hline España & 4 & $9.3 \%$ & $2013,2015,2018,2019$ \\
\hline Irán & 3 & $6.9 \%$ & $2000,2019,2020$. \\
\hline Finlandia & 3 & $6.9 \%$ & 2014, 2015, 2016. \\
\hline Mediterráneo oriental & 3 & $6.9 \%$ & 2017, 2018, 2020. \\
\hline Reino unido & 2 & $4.6 \%$ & 2016, 2019. \\
\hline Estados Unidos & 1 & $2.3 \%$ & 2016. \\
\hline Italia & 1 & $2.3 \%$ & 2016. \\
\hline Grecia & 1 & $2.3 \%$ & 2015. \\
\hline Etiopia & 1 & $2.3 \%$ & 2015. \\
\hline Inglaterra & 1 & $2.3 \%$ & 2018. \\
\hline Brasil & 1 & $2.3 \%$ & 2019. \\
\hline Corea del Sur & 1 & $2.3 \%$ & 2016. \\
\hline Holanda & 1 & $2.3 \%$ & 2016. \\
\hline Venezuela & 1 & $2.3 \%$ & 2011. \\
\hline Turquía & 1 & $2.3 \%$ & 2015. \\
\hline Portugal & 1 & $2.3 \%$ & 2020. \\
\hline Argentina & 1 & $2.3 \%$ & 2017. \\
\hline Países bajos & 1 & $2.3 \%$ & 2015. \\
\hline Noruega & 1 & $2.3 \%$ & 2017. \\
\hline Sudáfrica & 1 & $2.3 \%$ & 2020. \\
\hline Japón & 1 & $2.3 \%$ & 2007. \\
\hline Australia & 1 & $2.3 \%$ & 2003. \\
\hline Francia & 1 & $2.3 \%$ & 2013. \\
\hline
\end{tabular}

Fuente: elaboración propia (2020).

Signos, Investigación en Sistemas de Gestión

ISSN: 2145-1389 | e-ISSN: 2463-1140 | DOI: https://doi.org/10.15332/24631140

Vol. 13 N.० 2 | julio-diciembre de 2021 


\section{Conceptualización de eficiencia y productividad en salud}

Con base en la literatura se encuentran diferentes planteamientos como el propuesto por (Kämäräinen et ál., 2016), quien describe que debido a que no existe una definición uniforme de productividad y gracias a su estrecha relación con la eficiencia, la misma puede entenderse como eficiencia técnica; esto al inferir que al evaluar la eficiencia de un sistema o unidad se evidencia la capacidad con que los productos se convierten en insumos y, por ende, se estaría evaluando la productividad. Por su parte, Seddighi et ál. (2020) plantea que el concepto de eficiencia se equipara o iguala con el de productividad, y señala que el resultado final es la medición del rendimiento, y que el valor de la entrada se evalúa mediante la salida. Sin embargo, autores como Parra (2012) hacen mayor énfasis en dichos conceptos y su abordaje en el ámbito sanitario, y señalan que la eficiencia consiste en verificar la incidencia en la mejora de la salud de la población con el mejor uso de los recursos posibles, es decir, la forma adecuada de usar los recursos para lograr una mejoría en el estado de salud del paciente. Tomando como ejemplo un usuario oncológico, se puede decir que el sistema es eficiente cuando posterior a su tratamiento el paciente presenta mejoría en su estado de salud, lo cual se podrá evidenciar con la medición de marcadores y células tumorales, exámenes sanguíneos, de imagen y físicos sin alteraciones. Por su parte, la productividad en los servicios de salud ha sido concebida como la relación entre los eventos de servicios efectivos y los resultados esperados con los servicios prestados, entendiendo la productividad como la actividad desarrollada por la IPS y en la cual, además de integrar el uso adecuado y eficiente de los recursos, se aborda el resultado esperado con los servicios prestados y su impacto en la sociedad. Para el ejemplo del paciente oncológico, se espera que posterior a su tratamiento y además de presentar mejoría en los parámetros de medición de las diversas alteraciones en su salud, el 
paciente pueda integrarse a la sociedad, desarrollar sus actividades de forma normal como cualquier individuo, gozar de un estado mental, social y físico saludable.

En este sentido, la conexión entre salud y productividad y su relevancia dentro de una estrategia empresarial, se demuestra cuando a medida que mejora el estado de salud, también lo hace la productividad (Schwartz y Riedel, 2010).

A continuación, en la figura 4 se ilustra el ejemplo de paciente oncológico y el abordaje de eficiencia y productividad en el sector salud. 
Figura 3. Relación eficiencia y productividad en los servicios de salud

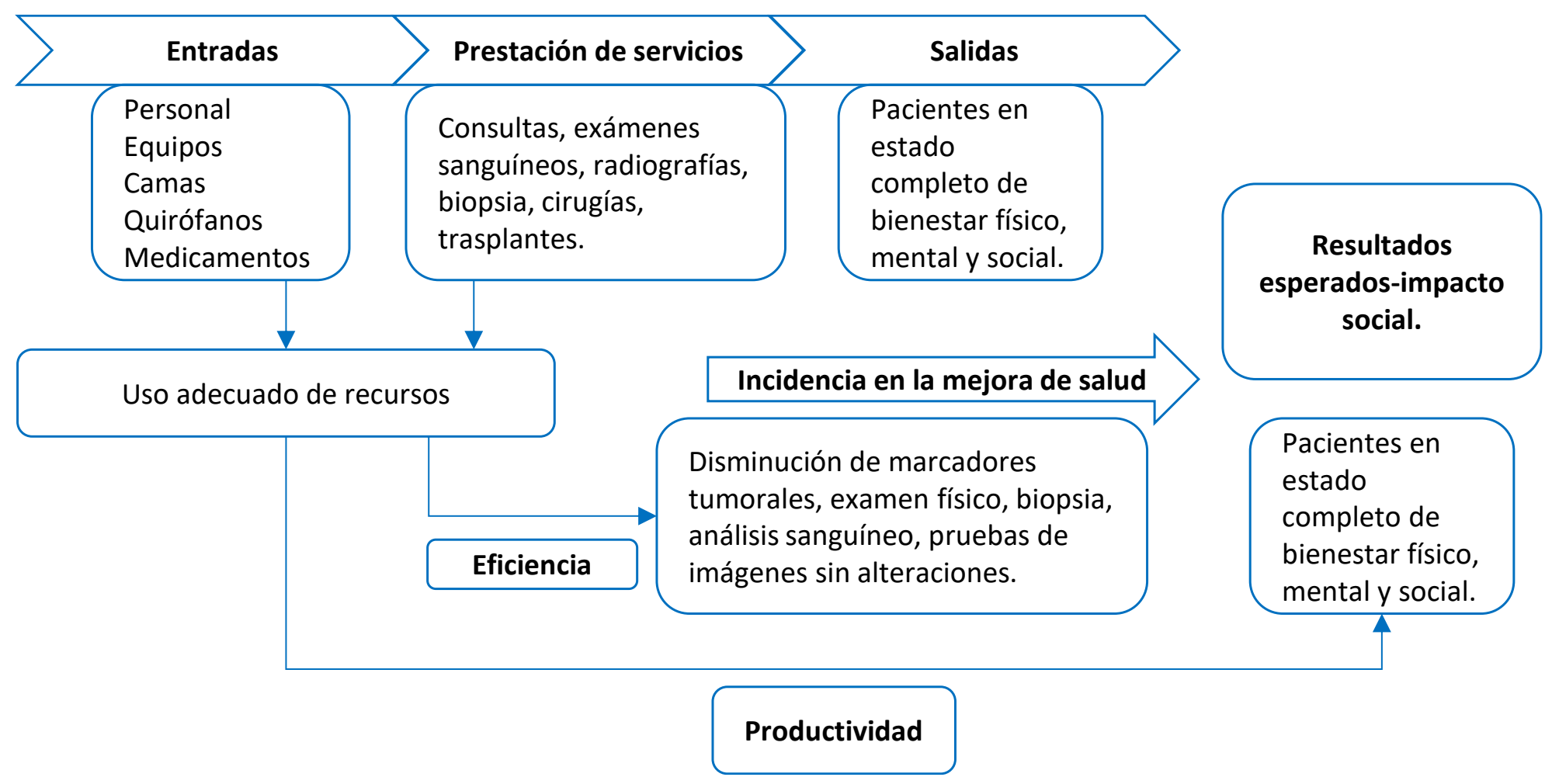

Fuente: elaboración propia (2020). 


\section{Variables de entrada y salida empleadas para la medición de la productividad en el sector salud}

Teniendo en cuenta que el sector salud tiene características relacionadas con aspectos de gran impacto para la medición de la productividad, tales como múltiples entradas y salidas, consumo colectivo de la salida y adhesión a principios de servicios públicos como continuidad, equidad e igualdad, definir las entradas y salidas es difícil y requiere de una gran precisión. Se debe tener en cuenta que al ser organizaciones prestadoras de servicios poseen características como la intangibilidad e interactividad en sus procesos, según lo describen Berhe et ál. (2017), por ende, la definición de variables atribuibles a entradas y salidas en los diferentes estudios de medición de la productividad en el ámbito sanitario se encuentra muy relacionada con el concepto imputado a la misma y el enfoque de cada investigador.

Algunos estudios como Giménez et ál. (2019), Herrero Tabanera et ál. (2015), Mitropoulos et ál. (2015), Del Rocío Moreno-Enguix et ál. (2018), Kämäräinen et ál. (2016), Hafidz et ál. (2018), Li et ál. 2017), Van Ineveld et ál. (2016), Charlesworth (2019), Ghahremanloo et ál. (2020), Kaya Samut y Cafrı (2016), Mancuso y Valdmanis (2016), Rocha et ál. (2020), Babalola y Moodley (2020), Li et ál. (2017), Lomas et ál. (2019), Nouraei Motlagh et ál. (2019), Liu et ál. (2019), Chai et ál. (2020), Francisco et ál. (2017), Nouraei Motlagh et ál. (2019), Flokou et ál. (2017), Bojke et ál. (2017) proponen el uso de variables de entrada y salida e indicadores de calidad mediante los cuales se estima la productividad de las instituciones basada en los productos obtenidos; sin embargo, otras investigaciones (Mohamadi et ál. (2020), Pourreza et ál. (2017), Masri y Asbu (2018), Estellita Lins et ál. (2019), Kim et ál. (2016), Rocha et ál. (2020), Liu et ál. (2019), Chai et ál. (2020), Seddighi et ál. (2020), definen variables de 
entrada y salida e indicadores de calidad en función de los resultados esperados en el sector salud o el impacto social causado tal y como se describe en la tabla 2. Dentro de las herramientas descritas para la evaluación de la calidad en las instituciones de salud, se encuentra el método de SERVQUAL que consistente en evaluar la percepción y expectativa del usuario y, en base a ello, determinar la calidad en los servicios de salud, como lo describe Ariza y Pardo (2018).

Tabla 2. Variables de entrada y salida utilizadas en el sector de la salud para la medición de la productividad

\begin{tabular}{|c|c|c|c|}
\hline 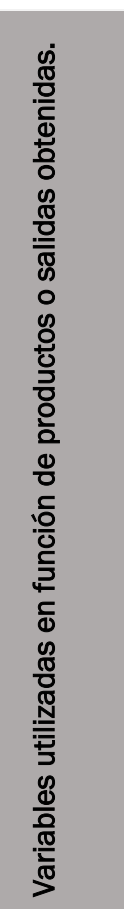 & $\begin{array}{c}\text { Variables de } \\
\text { salida }\end{array}$ & $\begin{array}{l}\text { Gastos totales, insumos de capital, } \\
\text { depreciación de equipos, salarios, } \\
\text { compra de medicamentos, camas, } \\
\text { equipos médicos, medicamentos, } \\
\text { equipo médico, enfermeras, número } \\
\text { de procedimientos realizados, datos de } \\
\text { hospitalización. } \\
\text { Altas hospitalarias, consultas externas, } \\
\text { consultas de urgencias, } \\
\text { procedimientos de cirugías } \\
\text { ambulatorias, tasa de ocupación y tasa } \\
\text { de rotación, visitas ambulatorias. } \\
\text { Acceso al servicio, satisfacción del } \\
\text { paciente, seguimiento y prevención del } \\
\text { riesgo, limpieza del centro sanitario, } \\
\text { minutos de espera en sala de } \\
\text { urgencias, reingreso en los siguientes } \\
20 \text { días, pacientes remitidos a otro } \\
\text { nivel e infecciones nosocomiales, } \\
\text { acceso a los servicios, idoneidad en la } \\
\text { atención, número real de camas } \\
\text { disponibles, paciente satisfecho, } \\
\text { espera total en días, total minutos de } \\
\text { espera en urgencias. }\end{array}$ & $\begin{array}{l}\text { Giménez et ál. (2019), Herrero } \\
\text { Tabanera et ál. (2015), } \\
\text { Mitropoulos et ál. (2015), Del } \\
\text { Rocío Moreno-Enguix et ál. } \\
\text { (2018), Kämäräinen et ál. } \\
\text { (2016), Hafidz et ál. (2018), Li } \\
\text { et ál. 2017), Van Ineveld et ál. } \\
\text { (2016), Charlesworth (2019), } \\
\text { Ghahremanloo et ál. (2020), } \\
\text { Kaya Samut y Cafrı (2016), } \\
\text { Mancuso y Valdmanis (2016), } \\
\text { Rocha et ál. (2020), Babalola y } \\
\text { Moodley (2020), Li et ál. } \\
\text { (2017), Lomas et ál. (2019), } \\
\text { Nouraei Motlagh et ál. (2019), } \\
\text { Liu et ál. (2019), Chai et ál. } \\
\text { (2020), Francisco et ál. (2017), } \\
\text { Nouraei Motlagh et ál. (2019), } \\
\text { Flokou et ál. (2017), Bojke et ál. } \\
\text { (2017). }\end{array}$ \\
\hline 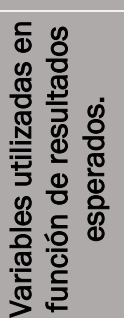 & $\begin{array}{c}\text { Variables de } \\
\text { salida } \\
\text { Indicadores } \\
\text { de calidad }\end{array}$ & $\begin{array}{l}\text { Número de médicos, enfermeras, } \\
\text { parteras, camas del hospital por } 1000 \\
\text { habitantes. } \\
\text { Esperanza de vida al nacer, mortalidad } \\
\text { infantil en menores de } 1 \text { año y } 5 \text { años. } \\
\text { Seguimiento y prevención del riesgo, } \\
\text { remisión de pacientes, reingreso. }\end{array}$ & $\begin{array}{l}\text { Mohamadi et ál. (2020), } \\
\text { Pourreza et ál. (2017), Masri y } \\
\text { Asbu (2018), Estellita Lins et ál. } \\
\text { (2019), Kim et ál. (2016), } \\
\text { Rocha et ál. (2020), Liu et ál. } \\
\text { (2019), Chai et ál. (2020), } \\
\text { Seddighi et ál. (2020). }\end{array}$ \\
\hline
\end{tabular}

Fuente: elaboración propia (2020). 
Por lo tanto, las entradas y salidas definidas para la medición de la productividad en el ámbito sanitario están ligadas a las dimensiones y servicios a evaluar. Chai et ál. (2020) señala que las entradas, salidas y fuentes de datos para la medición de la productividad se realizan según las entradas se transforman eficientemente en salidas y tienen un balance entre el número de entradas con respecto a las salidas. Tanto en las entradas como en las salidas se pueden tomar indicadores propios de las instituciones para realizar estos tipos de medición y evaluación de la productividad vs. la eficiencia. Lomas et ál. (2019) toman como entradas los factores económicos que influyen directamente en la operación de los servicios de salud del Reino Unido, sin tener en cuenta factores externos; dentro de las entradas para el estudio de la medición de productividad, se encuentran factores de costos inevitables como la atención hospitalaria, ambulatoria y comunitaria.

\section{Dimensiones abordadas en el sector salud para la medición de la productividad}

Con base en el análisis bibliográfico realizado la medición de la productividad se puede realizar empleando abordaje de tres grandes dimensiones descritas en la tabla 3. 
Tabla 3. Dimensiones abordadas en el sector salud para medición de la productividad

\begin{tabular}{|c|c|}
\hline Dimensión & $\begin{array}{c}\text { Descripción } \\
\text { Financiera }\end{array}$ \\
$\begin{array}{c}\text { En la cual se analiza o evalúa la inversión del capital que realizan las instituciones } \\
\text { con el fin de que sus procesos se lleven a cabo (amortizaciones, suministros, compra } \\
\text { de bienes, salarios). }\end{array}$ \\
\hline Física & $\begin{array}{r}\text { En donde se analizan los insumos o elementos físicos con los que cuenta la } \\
\text { organización para el desarrollo de actividades o procesos (número de camas de } \\
\text { hospitalización, exámenes, complejidad de los servicios). }\end{array}$ \\
\hline $\begin{array}{r}\text { Talento } \\
\text { Humano }\end{array}$ & $\begin{array}{r}\text { Número de profesionales en salud y administrativos de los que dispone la entidad } \\
\text { para el logro de objetivos o resultados esperados. }\end{array}$ \\
\hline
\end{tabular}

Fuente: Elaboración propia (2020).

Dado que la prestación de los servicios de salud se caracteriza por disponer de múltiples insumos para generar múltiples productos, el abordaje de las tres dimensiones descritas ha demostrado ser el análisis más utilizado para evaluación de la productividad; en este se abordan diferentes factores que influyen en la generación del resultado esperado en el ámbito sanitario, se considera la inversión del capital, los insumos físicos y el talento humano, y a partir estos se formulan las variables de medición (entradas y salidas). Rocha et ál. (2020) plantean que, para la medición de la productividad, las perspectivas socio económicas como el ausentismo laboral, los días de hospitalización y la muerte prematura, entre otras, son esenciales para evaluar los costos asociados a la atención por hospitalizaciones evitables y años de vida potencial perdidos.

Metodologías de medición de la productividad en el sector de la salud

Diferentes métodos para la medición de la productividad se han propuesto actualmente, en lo que se encuentran desde herramientas simples de sencilla aplicación y modelos sofisticados desarrollados a partir de la combinación de varios instrumentos. Varios autores plantean que, dentro de los métodos de medición convencionales se encuentran los basados en índices (índice de Malmquist e indicador de Luenberger), considerados los 
más simples y comunes, y los métodos basados en análisis de frontera, dentro de los cuales se hallan el DEA y análisis de frontera estocástica (Djellal y Gallouj, 2013; Estellita Lins et ál., 2019; Orozco-Gallo, 2014).

Los métodos basados en índices han sido tradicionalmente utilizados para la medición de la productividad en diferentes sectores, el índice de Malmquits ha sido el que mayor incidencia ha presentado en la literatura, basado en el cálculo de cocientes de funciones de distancia que permite medir el cambio productivo de la organización (crecimiento de productividad total de los factores), así como descomponer este crecimiento en cambio técnico y cambio de eficiencia técnica, según lo describen Li et ál. (2017a). Por su parte, el indicador de Luenberger ha adquirido recientemente importancia en el análisis de la productividad, aunque con escasa literatura aún, y según Orozco-Gallo (2014) presenta ventajas ya que su noción menos restrictiva permite evaluar cambios en los productos e insumos de forma simultánea y medir el nivel total de la productividad a partir de los niveles alcanzados por cada unidad productiva.

Dentro de los métodos de frontera mencionados, el más utilizado para la medición de la productividad actualmente es el DEA, caracterizado por ser un método no paramétrico mediante el cual es posible construir una frontera tecnológica con unidades productivas, las cuales usan las mejores prácticas, escogen una orientación especifica hacia el insumo o producto e identifican el máximo o mínimo nivel de producto alcanzable con cierta combinación de insumos (Kämäräinen et ál., 2016; Kaya Samut y Cafrı, 2016). Por su parte, la frontera estocástica (FE) ha sido utilizada más recientemente, aunque en menor proporción, ya que, según los describe Hafidz et ál. (2018), puede abordar mejor el ruido presente en los datos, pero requiere para su uso unos supuestos sobre la forma funcional y la distribución de errores. 
Nouraei Motlagh et ál. (2019) indican que para estudios retrospectivos con diseños descriptivos y analíticos se emplea el método DEA, utilizado ampliamente para medir la eficiencia relativa y la productividad; aunque el índice de Malmquist es también uno de los modelos más significativos en medición y análisis de factores que afectan la productividad.

Ghahremanloo et ál. (2020) describen el DEA como una técnica basada en programaciones lineales que calcula diferentes variables de rendimiento, estudia el insumo de entrada, tiene en cuenta el número de trabajadores de la salud, así como otros empleados, y el número de camas de pacientes, mientras que las salidas las toma de la tasa de ocupación de camas y tasa de rotación.

Adicionalmente, dentro de las metodologías de medición de la productividad, algunos estudios se han basado en determinar la relación entre eficiencia y calidad al usar indicadores mediante los cuales se pueda establecer la relación directa en cada uno de los procesos o unidades productivas a evaluar. Así encontraron que existe una clara relación entre ambas y se ve afectada la calidad en aquellos procesos que requieren mayor tiempo para su desarrollo, según lo plantea Laine et ál. (2005). Bojke et ál. (2017) proponen medir la productividad calculando el crecimiento de la productividad total de los factores (TFP) entre dos periodos de tiempo, los cuales hacen una medición al observar el número de entras y salidas en un tiempo inicial y evaluarlas en un tiempo posterior.

En la tabla 4 se resumen las metodologías descritas y las dimensiones abordadas en la literatura analizada. 
Tabla 4. Metodologías empleadas para la medición de la productividad en el sector de la salud

\begin{tabular}{|c|c|c|c|}
\hline Metodologías propuestas & En combinación & Dimensiones abordadas & Autores \\
\hline \multirow{9}{*}{ Análisis envolvente de datos (DEA) } & $\begin{array}{l}\text { Boostrapping. } \\
\text { Análisis discriminante. } \\
\text { Procedimiento de análisis de } \\
\text { componentes principales (PCA). }\end{array}$ & Financiero, físico, talento humano. & Del Rocío Moreno-Enguix et ál. (2018). \\
\hline & Análisis Tobit. & Financiera. & Aragon Aragon et ál. (2017); Kaya Samut y Cafı́, (2016). \\
\hline & $\begin{array}{l}\text { Uso de indicadores y analisis } \\
\text { discriminante. }\end{array}$ & Financiero, talento humano. & Caballer-Tarazona et ál., (2010). \\
\hline & Técnica Bayesiana. & Financiera, físico, talento humano. & Mitropoulos et ál. (2015). \\
\hline & Índice de Malmquist. & Financiera, físico, talento humano. & $\begin{array}{l}\text { Herrero Tabanera et ál. (2015), Mohamadi et ál. (2020), Li } \\
\text { et ál. (2017), Masri y Asbu (2018), Kim et ál. (2016), Kaya } \\
\text { Samut y Cafrı (2016), Babalola y Moodley (2020), Gómez } \\
\text { Gallego et ál. (2019), Díaz y Gómez (2013), Hollingsworth } \\
\text { (2003), Anthun et ál. (2017), Cheng et ál. (2016), Liu et ál. } \\
\text { (2019), Mancuso y Valdmanis (2016), Chai et ál. (2020). }\end{array}$ \\
\hline & DEA-EEP. & Físico y financiero. & $\begin{array}{l}\text { Pourreza et ál. (2017), Van Ineveld et ál. (2016b), Highfill y } \\
\text { Ozcan (2016), Ghahremanloo et âl. (2020). }\end{array}$ \\
\hline & Mapas conceptuales. & Financiero, físico. & Estellita Lins et ál. (2019). \\
\hline & Frontera Estocástica. & Financiero, físico, talento humano. & Hafidz et ál. (2018), Kämäräinen et ál. (2016). \\
\hline & Indicador de Luenberger. & Financiero, físico, talento humano. & Giménez et ál. (2019). \\
\hline $\begin{array}{l}\text { Metodología basada en el análisis } \\
\text { discriminante multivariado. }\end{array}$ & & Financiero, físico, talento humano. & Fontalvo Herrera (2012). \\
\hline Índice de Malmquist. & Boostrapping y frontera estocástica. & Físico, talento humano. & Nghiem et ál. (2011) \\
\hline $\begin{array}{l}\text { Metodología basada en las mejores } \\
\text { prácticas de gestión. }\end{array}$ & $\begin{array}{c}\text { Fase cualitativa (método VIPLAN) y } \\
\text { cuantitativa (variables económicas, } \\
\text { técnicas, clínicas). }\end{array}$ & Financiero, técnico, clínico. & Parra Riveros, (2012). \\
\hline
\end{tabular}

Signos, Investigación en Sistemas de Gestión

ISSN: 2145-1389 | e-ISSN: 2463-1140 | DOI: https://doi.org/10.15332/24631140

Vol. 13 N. ${ }^{\circ} 2$ | julio-diciembre de 2021 
Conjunción del Manual FIM:

productividad, el modelo ntegral de

dirección estratégica de Fred David.

Uso de indicadores de estructura,

proceso y resultado.

Metodología basada en el uso inverso del

indice de productividad y función de

distancia direccional, para medir e

efecto de los insumos. ineficaces 0

productos no conformes.

Matriz de indicadores.

Análisis estadístico y análisis de

$$
\text { sensibilidad. }
$$

Variables socioeconómicas y

demográficas, estrategias

$$
\text { econométricas. }
$$

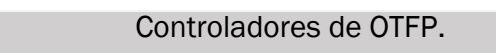

Controladores de OTFP.

en Super-

$$
\text { Slack (SBM). }
$$

Análisis Kendrick-Creamer y coeficiente

$$
\text { Anderson-Peterson. }
$$

Financiero, físico, talento humano.

Berhe et ál. (2017), Watanabe y Tanaka (2007).

Fuente: elaboración propia (2020).

Signos, Investigación en Sistemas de Gestión

ISSN: 2145-1389 | e-ISSN: 2463-1140 | DOI: https://doi.org/10.15332/24631140

Vol. 13 N. ${ }^{\circ} 2$ | julio-diciembre de 2021 


\section{Conclusiones}

Se encuentra una mayor producción literaria sobre la medición de la productividad en el sector de la salud entre los años 2015 a 2020, 4 veces mayor a la encontrada entre los años 2010 a 2014, lo cual evidencia que el interés de la comunidad científica en dicho tema ha incrementado en los últimos años.

Producto de la revisión bibliográfica realizada, fue posible analizar el concepto que el punto de vista clínico ha dado tanto a la eficiencia como a la productividad, con el fin de lograr su medición. Así, se entiende la eficiencia como el mejor uso posible de los insumos y su incidencia en la mejora del estadio de salud de cada paciente; por su parte, la productividad es concebida como la relación existente entre los insumos o entradas disponibles para la prestación de los servicios en salud y cómo esto repercute en los resultados esperados y el impacto social causado. Por lo anterior al entender que los servicios de salud utilizan múltiples insumos para generar múltiples productos y que para calcular la productividad se requiere identificar tanto entradas como salidas, es necesario que por cada área o servicio a evaluar en las IPS se definan adecuadamente aquellas unidades productivas que logren representar el crecimiento o decrecimiento de la productividad con el fin de obtener una estimación real de la misma; también se debe considerar el abordaje de las diferentes dimensiones (financiera, física, talento humano) que influyen en la estimación de la productividad e integran el sistema de la organización, lo cual facilita así la evaluación total de la productividad. Diferentes métodos para la medición de la productividad se han descrito, la variabilidad en su uso ha sido atribuida a las ventajas y desventajas analizadas en los diferentes estudios; por tanto, la combinación de dos o más herramientas de medición ha demostrado tener mayor impacto en la 
estimación de la productividad en IPS. Dentro de los métodos descritos, el DEA es la herramienta más utilizada actualmente para la medición de la eficiencia, ya que permite analizar la mayoría de las variables disponibles dentro de la organización sin tener un limitante de tiempo. No obstante, aunque sea uno de los más empleados en la evaluación de la eficiencia en servicios de salud, este presenta limitaciones metodológicas y alta inferencia estadística, por lo cual es necesario apoyarse con otros métodos de medición tanto econométricos como no econométricos, gracias a los cuales se puede minimizar la inferencia estadística. Uno de estos métodos es el índice de Malmquits, que logra con esta combinación calcular la función de distancia y posteriormente evaluar el cambio de la productividad (crecimiento de la productividad total de los factores) en diferentes periodos de tiempo, así mismo, descomponer el crecimiento de productividad para determinar el cambio técnico y de eficiencia en las organizaciones.

Otras técnicas utilizadas son el análisis de componentes principales (PCA), el boostrapping y el análisis discriminante, gracias a las cuales se permite seleccionar indicadores para lograr la supervisión de una gestión más simple y operativa en un sistema sanitario determinado y detectar unidades influyentes que determina el resultado. La técnica bayesiana también ha demostrado ser una nueva tendencia que permite disminuir la inferencia estadística en este tipo de estudios.

La aplicación del DEA basado en variables cuantitativas obtenidas mediante el uso de mapas conceptuales se ha descrito como una nueva metodología en la cual se tiene en cuenta la gestión cualitativa y los métodos cuantitativos para estimar la productividad en las organizaciones de salud. Otras metodologías se han basado en el uso de indicadores de productividad y evaluación por áreas en cada una de las organizaciones, sin utilización de herramientas econométricas, en donde, tras el análisis de 
indicadores y la recolección de información mediante entrevistas, se ha estimado la productividad en las IPS.

Además, se han integrado los indicadores de calidad a la medición de la productividad con el fin de establecer la relación existente entre ambas, basados en la concepción de que para incrementar la calidad se requiere la inversión de capital, lo cual afectaría la productividad, mientras que la mejora de la productividad y la disminución de los costos afecta la calidad. Se encuentra así una estrecha relación entre calidad y eficiencia en el sector de la salud, en donde aquellos servicios que requieren mayor tiempo para su desarrollo son los que han demostrado tener mayor afectación de la calidad.

Por lo tanto, al momento de seleccionar la combinación de instrumentos de medición, las áreas a evaluar y los productos o resultados a medir en las IPS deben ser tenidas en cuenta consideraciones como la disponibilidad de la información, las variables de entradas y salidas establecidas, las desventajas atribuidas a los diferentes métodos de medición como inferencia estadística, los requerimientos de supuesto de forma funcional, las restricción para uso en sistemas con múltiples entradas y salidas, los indicadores de medición de calidad o demás.

Es importante señalar que los métodos utilizados para la medición de la productividad en el sector de la salud se han basado en la relación producto/insumo, de acuerdo a las variables empleadas y áreas de evaluación seleccionadas; sin embargo, nuevos estudios proponen que para la medición de la productividad se deben tener en cuenta los productos no conformes o insumos ineficaces, que también afectan la productividad de las organizaciones. Así, esta es una nueva línea de investigación que busca una aproximación más precisa de productividad en las organizaciones. 


\section{Referencias}

Anthun, K. S., Kittelsen, S. A. C., y Magnussen, J. (2017). Productivity growth, case mix and optimal size of hospitals. A 16-year study of the Norwegian hospital sector. Health Policy, 121(4), 418-425. https://doi.org/10.1016/j.healthpol.2017.01.006

Aragon Aragon, M. J., Castelli, A., y Gaughan, J. (2017). Hospital Trusts productivity in the English NHS: Uncovering possible drivers of productivity variations. PLOS ONE, 12(8), e0182253. https://doi.org/10.1371/journal.pone.0182253

Ariza, G. Y. G., y Pardo, Y. A. (2018). Importancia de la calidad en los servicios de salud y Servqual como método para la medición de la calidad en los servicios de salud [tesis de especialización]. Universidad de Santander.

Babalola, T. K., y Moodley, I. (2020). Technical efficiency and productivity of public district hospitals in KwaZulu-Natal province, South Africa. Journal of Public Health Research, 9(1). https://doi.org/10.4081/jphr.2020.1741

Berhe, E., Abebe, B., y Azene, D. (2017). A new perspective to productivity measurement. Total Quality Management \& Business Excellence, 28(1-2), 205-217. https://doi.org/10.1080/14783363.2015.1053804

Bojke, C., Castelli, A., Grašič, K., y Street, A. (2017). Productivity Growth in the English National Health Service from 1998/1999 to 2013/2014. Health Economics, 26(5), 547-565. https://doi.org/10.1002/hec.3338

Bustos, A. D. B. (2017). Análisis de la productividad de los servicios de salud y su articulación con la viabilidad financiera del hospital Juan Hernando Urrego E.S.E única IPS pública del municipio de Aguazul Casanare [tesis de especialización]. Universidad Nacional Abierta y a Distancia.

Caballer-Tarazona, M., Moya-Clemente, I., Vivas-Consuelo, D., y Barrachina-Martínez, I. (2010). A model to measure the efficiency of hospital performance. Mathematical and Computer Modelling, 52(7-8), 1095-1102. https://doi.org/10.1016/j.mcm.2010.03.006

Chai, P., Zhang, Y., Zhou, M., Liu, S., y Kinfu, Y. (2020). Health system productivity in China: A comparison of pre- and post-2009 healthcare reform. Health Policy and Planning, 35(3), 257-266. https://doi.org/10.1093/heapol/czz157

Signos, Investigación en Sistemas de Gestión ISSN: 2145-1389 | e-ISSN: 2463-1140 | DOI: https://doi.org/10.15332/24631140 Vol. 13 N.o 2 | julio-diciembre de 2021 
Charlesworth, A. (2019). Measuring the productivity of the health care system: The experience of the United Kingdom by Anita Charlesworth. OECD Journal on Budgeting, 19(3). https://doi.org/10.1787/75a91975-en

Cheng, Z., Cai, M., Tao, H., He, Z., Lin, X., Lin, H., y Zuo, Y. (2016). Efficiency and productivity measurement of rural township hospitals in China: A bootstrapping data envelopment analysis. BMJ Open, 6(11), e011911. https://doi.org/10.1136/bmjopen-2016-011911

Del Rocío Moreno-Enguix, M., Gómez-Gallego, J. C., y Gómez Gallego, M. (2018). Analysis and determination the efficiency of the European health systems. The International Journal of Health Planning and Management, 33(1), 136-154. https://doi.org/10.1002/hpm.2412

Díaz, A. S., y Gómez, G. I. (2013). Evolución de la productividad y asociación con la satisfacción en la atención hospitalaria y especializada de los sistemas sanitarios de las Comunidades Autónomas. Investigaciones regionales, 27, 7-32. https://www.redalyc.org/articulo.oa?id=28928823001

Djellal, F., y Gallouj, F. (2013). The productivity challenge in services: Measurement and strategic perspectives. The Service Industries Journal, 33(3-4), 282-299. https://doi.org/10.1080/02642069.2013.747519

Estellita Lins, M. P., Netto, S. O. A., y de Castro Lobo, M. S. (2019). Multimethodology applied to the evaluation of Healthcare in Brazilian municipalities. Health Care Management Science, 22(2), 197-214. https://doi.org/10.1007/s10729-018-9432-Z

Flokou, A., Aletras, V., y Niakas, D. (2017). A window-DEA based efficiency evaluation of the public hospital sector in Greece during the 5-year economic crisis. PLOS ONE, 12(5), e0177946. https://doi.org/10.1371/journal.pone.0177946

Fontalvo Herrera. (2012). Evaluación de la productividad de las entidades prestadoras de servicios de salud (EPS) del régimen subsidiado en Colombia, por medio del analisis discriminante. Hacia la Promoción de la Salud, 17(2), 60-78. http://www.scielo.org.co/pdf/hpsal/v17n2/v17n2a05.pdf

Francisco, O., Marco, D. y Cecilia, M. L. (2017). Indicadores de productividad en hospitales públicos. Revista Científica "Visión de Futuro", 21(2), 40-60. https://www.redalyc.org/pdf/3579/357955446002.pdf 
Ghahremanloo, M., Hasani, A., Amiri, M., Hashemi-Tabatabaei, M., KeshavarzGhorabaee, M., y Ustinovičius, L. (2020). A novel DEA model for hospital performance evaluation based on the measurement of efficiency, effectiveness, and productivity. Engineering Management in Production and Services, 12(1), 7-19. https://doi.org/10.2478/emj-2020-0001

Giménez, V., Prieto, W., Prior, D., \& Tortosa-Ausina, E. (2019a). Evaluation of efficiency in Colombian hospitals: An analysis for the post-reform period. Socio-Economic Planning Sciences, 65, 20-35. https://doi.org/10.1016/j.seps.2018.02.002

Gómez Gallego, J. C., García García, J. F., y Gómez Gallego, M. (2019). Eficiencia y productividad de los sistemas de salud de los países de la Unión Europea. Estudios de Economía Aplicada, 37(2), 196. https://doi.org/10.25115/eea.v37i2.2613

Hafidz, F., Ensor, T., y Tubeuf, S. (2018). Efficiency Measurement in Health Facilities: A Systematic Review in Low- and Middle-Income Countries. Applied Health Economics and Health Policy, 16, 465-480. https://doi.org/10.1007/s40258-018$\underline{0.385-7}$

Herrero Tabanera, L., Martín Martín, J. J., y López del Amo González, M. del P. (2015). Eficiencia técnica de los hospitales públicos y de las empresas públicas hospitalarias de Andalucía. Gaceta Sanitaria, 29(4), 274-281.

https://doi.org/10.1016/j.gaceta.2015.03.001

Highfill, T., y Ozcan, Y. (2016). Productivity and quality of hospitals that joined the Medicare Shared Savings Accountable Care Organization Program. International Journal of Healthcare Management, 9(3), 210-217. https://doi.org/10.1179/2047971915Y.0000000020

Hollingsworth, B. (2003). Non-Parametric and Parametric Applications Measuring Efficiency in Health Care. Health Care Management Science, 6, 203-218. https://doi.org/10.1023/a:1026255523228

Kämäräinen, V. J., Peltokorpi, A., Torkki, P., y Tallbacka, K. (2016). Measuring healthcare productivity - from unit to system level. International Journal of Health Care Quality Assurance, 29(3), 288-299. https://doi.org/10.1108/IJHCQA-04-2015$\underline{0050}$ 
Kaya Samut, P., y Cafrı, R. (2016). Analysis of the Efficiency Determinants of Health Systems in OECD Countries by DEA and Panel Tobit. Social Indicators Research, 129(1), 113-132. https://doi.org/10.1007/s11205-015-1094-3

Kim, Y., Oh, D., y Kang, M. (2016). Productivity changes in OECD healthcare systems: Bias-corrected Malmquist productivity approach. The International Journal of Health Planning and Management, 31(4), 537-553.

https://doi.org/10.1002/hpm.2333

Laine, J., Finne-Soveri, U. H., Björkgren, M., Linna, M., Noro, A., y Häkkinen, U. (2005). The association between quality of care and technical efficiency in long-term care. International Journal for Quality in Health Care, 17(3), 259-267. https://doi.org/10.1093/intqhc/mzio32

Li, N.-N., Wang, C.-H., Ni, H., y Wang, H. (2017). Efficiency and Productivity of Countylevel Public Hospitals Based on the Data Envelopment Analysis Model and Malmquist Index in Anhui, China: Chinese Medical Journal, 13o(23), 2836-2843. https://doi.org/10.4103/0366-6999.219148

Liu, W., Xia, Y., y Hou, J. (2019). Health expenditure efficiency in rural China using the super-SBM model and the Malmquist productivity index. International Journal for Equity in Health, 18(1), 111. https://doi.org/10.1186/s12939-019-1003-5

Lomas, J., Martin, S., y Claxton, K. (2019). Estimating the Marginal Productivity of the English National Health Service From 2003 to 2012. Value in Health, 22(9), 9951002. https://doi.org/10.1016/j.jval.2019.04.1926

Luna Cardozo, M. (2011). Instrumento para la medición de la productividad en instituciones públicas del sector salud. Universidad Nacional Experimental Politécnica, 1(2). http://redip.bqto.unexpo.edu.ve

Magnussen, J., y Nyland, K. (2008). Measuring efficiency in clinical departments. Health Policy, 87(1), 1-7. https://doi.org/10.1016/j.healthpol.2007.09.013

Mancuso, P., y Valdmanis, V. G. (2016). Care Appropriateness and Health Productivity Evolution: A Non-Parametric Analysis of the Italian Regional Health Systems. Applied Health Economics and Health Policy, 14(5), 595-607. https://doi.org/10.1007/s40258-016-0257-y 
Masri, M. D., y Asbu, E. Z. (2018). Productivity change of national health systems in the WHO Eastern Mediterranean region: Application of DEA-based Malmquist productivity index. Global Health Research and Policy, 3(1). https://doi.org/10.1186/s41256-018-0077-8

Mitropoulos, P., Talias, M. A., y Mitropoulos, I. (2015). Combining stochastic DEA with Bayesian analysis to obtain statistical properties of the efficiency scores: An application to Greek public hospitals. European Journal of Operational Research, 243(1), 302-311. https://doi.org/10.1016/j.ejor.2014.11.012

Mohamadi, E., Olyaee Manesh, A., Takian, A., Majdzadeh, R., Hosseinzadeh Lotfi, F., Sharafi, H., Jowett, M., Kiani, M. M., Hosseini Qavam Abadi, L., Fazaeli, A. akbar, Goodarzi, Z., Sajadi, H. S., Noori Hekmat, S., y Freidoony, L. (2020). Technical efficiency in health production: A comparison between Iran and other upper middle-income countries. Health Policy and Technology, 9(3). https://doi.org/10.1016/j.hlpt.2020.06.007

Nghiem, S., Coelli, T., y Barber, S. (2011). Sources of Productivity Growth in Health Services: A Case Study of Queensland Public Hospitals. Economic Analysis and Policy, 41(1), 37-48. https://doi.org/10.1016/S0313-5926(11)50003-7

Nouraei Motlagh, S., Ghasempour, S., Yusefzadeh, H., Lotfi, F., Astaraki, P., y Saki, K. (2019). Evaluation of the Productivity of Hospitals Affiliated to Lorestan University of Medical Sciences Using the Malmquist and the Kendrick-Creamer Indices. Shiraz E-Medical Journal, 2O(7). https://doi.org/10.5812/semj.85222

Orozco-Gallo, A. J. (2014). Una aproximación regional a la eficiencia y productividad de los hospitales públicos colombianos. Documentos de trabajo sobre economía regional. Banco de la República. https://doi.org/10.32468/dtseru.201

Parra Riveros, H. (2012). Metodología para la evaluación de la eficiencia integral en servicios de salud en instituciones públicas prestadoras de servicios de salud (IPS E.S.E) [tesis de maestría]. Universidad Nacional de Colombia.

Pourreza, A., Alipour, V., Arabloo, J., Bayati, M., y Ahadinezhad, B. (2017). Health production and determinants of health systems performance in WHO Eastern Mediterranean Region. Eastern Mediterranean Health Journal, 23(5), 368-374. https://doi.org/10.26719/2017.23.5.368 
Prokopenko, I. I. (1989). La gestion de la productividad: Manual práctico. Oficina Internacional del Trabajo.

Rocha, J. V. M., Marques, A. P., Moita, B., y Santana, R. (2020). Direct and lost productivity costs associated with avoidable hospital admissions. BMC Health Services Research, 2O(1). https://doi.org/10.1186/s12913-020-5071-4

Schwartz, S. M., y Riedel, J. (2010). Productivity and Health: Best Practices for Better Measures of Productivity: Journal of Occupational and Environmental Medicine, 52(9), 865-871. https://doi.org/10.1097/JOM.obo13e3181ed8686

Seddighi, H., Nosrati Nejad, F., y Basakha, M. (2020). Health systems efficiency in Eastern Mediterranean Region: A data envelopment analysis. Cost Effectiveness and Resource Allocation, 18. https://doi.org/10.1186/s12962-020-00217-9

Van Ineveld, M., van Oostrum, J., Vermeulen, R., Steenhoek, A., y van de Klundert, J. (2016). Productivity and quality of Dutch hospitals during system reform. Health Care Management Science, 19(3), 279-290. https://doi.org/10.1007/s10729-0159321-7

Watanabe, M., y Tanaka, K. (2007). Efficiency analysis of Chinese industry: A directional distance function approach. Energy Policy, 35(12), 6323-6331. https://doi.org/10.1016/j.enpol.2007.07.013

Yu, J., Liu, Z., Zhang, T., Hatab, A. A., y Lan, J. (2020). Measuring productivity of healthcare services under environmental constraints: Evidence from China. BMC Health Services Research, 2O(1), 673. https://doi.org/10.1186/s12913-020-05496-9 